\title{
Magnetresonanz-Tomografie in Echtzeit
}

(vorgetragen in der Plenarsitzung am 17. April 2009)

Jens Frahm, Shuo Zhang

„Bitte ruhig liegen bleiben und nicht bewegen!“ An eine solche Aufforderung werden sich die meisten derjenigen erinnern, die bereits einmal mit der Magnetresonanz-Tomografie (MRT) untersucht wurden; denn die Aufnahmen sind bewegungsempfindlich. Und doch berichtet diese Vorlage über das genaue Gegenteil: eine filmische MRT bewegter Organe in Echtzeit (Zhang et al. 2009).

Diese Diskrepanz zwischen radiologischer Praxis und wissenschaftlichem Fortschritt ist in der Tat überraschend. Denn seit der Erstbeschreibung der MRT als eines nichtinvasiven bildgebenden Verfahrens (Lauterbur 1973) hat sich deren technische, wissenschaftliche und klinische Weiterentwicklung als eine fortlaufende Erfolgsgeschichte herausgestellt, von der jedes Jahr weltweit nahezu 100 Millionen Menschen profitieren. Fundamentale Fortschritte betreffen neben der hochauflösenden und kontrastreichen Darstellung von krankhaften Gewebeveränderungen vielfältige weitergehende Untersuchungen, etwa des Gefäßsystems (Angiografie), des Stoffwechsels (lokalisierte Spektroskopie), der Hirnfunktionen (funktionelle MRT) und der Nervenfaserbahnen (Diffusionstensor-MRT). Grundlage dieser Fortschritte sind zwar auch technische Verbesserungen der Geräte, vor allem aber physikalische Erweiterungen der zugrundeliegenden Messverfahren wie die Einführung schneller Sequenzen in der Mitte der 1980er Jahre (Frahm et al. 1985). Bei genauer - retrospektiver - Betrachtung sind daher alle für die Realisierung einer Echtzeit-MRT wesentlichen Elemente seit vielen Jahren bekannt. Und es soll auch keinesfalls verschwiegen werden, dass vereinzelte Berichte bereits sehr dicht an diejenige Lösung herangekommen sind, die Shuo Zhang jetzt in seiner Göttinger Dissertation in überzeugender Qualität vorgelegt hat (Zhang 2009).

Das Konzept umfasst (i) eine schnelle und bewegungsresistente Messung einzelner Bilder, (ii) die fortlaufende Datenaufnahme einer Serie von Bildern ohne die Notwendigkeit einer Unterbrechung und (iii) die sofortige Rekonstruktion und unmittelbare Darstellung der Bilder einer Serie ohne spürbare zeitliche Verzögerung. Das Ziel ist die kontinuierliche Verfolgung 
eines dynamischen Prozesses mit ausreichender räumlicher und angemessener zeitlicher Auflösung. Solche Prozesse können von natürlichen Organbewegungen wie denen des Herzens bis zu der Kontrolle chirurgischer Interventionen beim Positionieren einer Biopsienadel oder eines Katheters reichen.

\section{Messtechnik}

Da die Überlegungen, die zur Echtzeit-MRT führen, offenkundig etwas mit dem Messprozess zu tun haben, ist es zunächst sinnvoll, das konventionelle Vorgehen zu beleuchten. In mathematischer Hinsicht entsprechen die Messdaten der MRT der Fourier-Transformierten des gewünschten Bildes. Mit anderen Worten: wenn die Messung eine angemessene Abtastung des Datenraumes liefert und diese Abtastung für ein zweidimensionales Bild auf einem rechtwinkligen Raster aus Datenpunkten erfolgt, dann besteht die Rekonstruktion des MRT-Bildes nur aus einer inversen FourierTransformation.

Um den Datenraum eines MRT-Bildes ausreichend dicht abzutasten, besteht der gesamte Messprozess in der Regel aus sehr vielen Einzelmessungen mit unterschiedlicher (eindimensionaler) Ortskodierung. Das konventionelle Verfahren tastet den Datenraum zeilenweise ab, wobei die gemessene Datenzeile bei jeder neuen Teilmessung in senkrechter Richtung um einen kleinen Betrag verschoben wird. Das sich ergebende rechtwinklige (kartesische) Datenmuster lässt sich dann sehr einfach zum Bild umrechnen.

Um trotz der notwendigen Wiederholungen von Teilmessungen eine kurze Messzeit sowie eine verzögerungsfreie Fortsetzung der Datenaufnahme für ein nachfolgendes Bild zu erzielen, kann bei der Wahl des physikalischen Messverfahrens für die Echtzeit-MRT vorzugsweise auf die fast low-angle shot (FLASH)-Technik zurückgegriffen werden. Erste dynamische Studien von Bewegungsvorgängen wurden daher schon vor über 20 Jahren realisiert (Frahm et al. 1986). Da die damaligen Versuche allerdings auf kartesisch kodierten Daten beruhten, ergab sich nur eine begrenzte räumliche und zeitliche Auflösung sowie eine eingeschränkte Bildqualität bei sehr schnellen Bewegungen. Das letztgenannte Problem hat entscheidend damit zu tun, dass das senkrechte Verschieben der frequenzkodierten Datenzeilen von Teilmessung zu Teilmessung durch einen phasenkodierenden Magnetfeldgradienten erfolgt. Auf diese Weise ist die Ortsinformation eines konventionellen MRT-Bildes einerseits in der Frequenz (Datenzeile: erste Dimension) und andererseits in der Phase der aufgenommenen Signale 
a

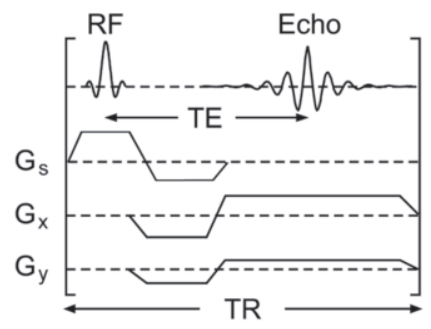

b

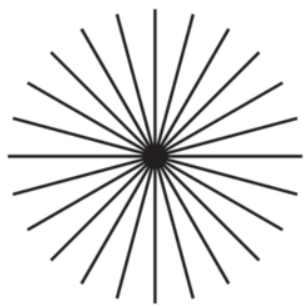

C

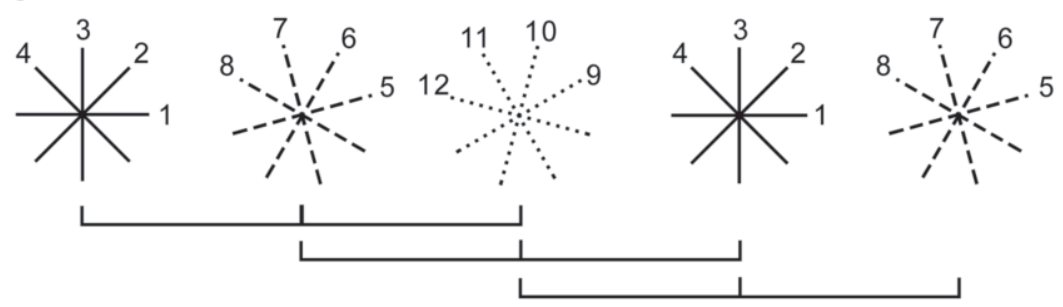

Abbildung 1: (a) FLASH MRT-Sequenz mit radialer Ortskodierung (Teilmessung): $\mathrm{RF}=$ Radiofrequenz-Anregungsimpuls, Echo = aufgezeichnetes Gradientenecho-Signal (Datenzeile), $\mathrm{TE}=$ Echozeit, $\mathrm{G}_{s}=$ schichtselektiver Magnetfeldgradient, $\mathrm{G}_{x}$ and $\mathrm{G}_{y}=$ variable frequenzkodierende Gradienten für die radiale Ortskodierung, TR = Repetitionszeit für die Wiederholung der Teilmessung mit unterschiedlicher Ortskodierung. (b) Radiale Abtastung des Datenraumes (Beispiel mit 12 Speichen). (c) Aufteilung einer radialen Abtastung mit 12 Speichen in 3 Umläufe mit je 4 Speichen. Derartige Varianten ermöglichen bei Messungen aufeinanderfolgender Bilder eine beschleunigte Bildrekonstruktion nach Erneuerung der Daten nur eines Umlaufes, also beispielsweise schon nach der Messung von nur 4 statt 12 Speichen eines nachfolgenden Bildes. Diese sliding window-Technikgestattet die Rekonstruktion von Bildserien mit erhöhter Bildfrequenz.

kodiert (senkrechte zweite Dimension). Während die Frequenzinformation während der sehr kurzen Teilmessung einer Datenzeile nicht ohne weiteres durch Bewegungen gestört werden kann, erweist sich die Phasenlage der Signale, die durch alle Teilmessungen definiert wird, als besonders anfällig. Die Verfälschung der ortskodierten Phasen durch ein während der Gesamtmessung bewegtes Objekt erzeugt bei der Berechnung des Bildes, die alle Phasen als Ortsinformation interpretiert, zum Teil erhebliche Fehler in der Form von Verschmierungen oder Geisterbildern.

Für die Entwicklung der Echtzeit-MRT wurde die kartesische Ortskodierung in der FLASH-Sequenz durch eine radiale Ortskodierung ersetzt. Dieser Ansatz beruht auf einer reinen Frequenzkodierung mit zwei orthogonalen Magnetfeldgradienten, die in aufeinanderfolgenden Teilmessun- 
gen des Bildes stets gemeinsam, aber mit unterschiedlicher Stärke geschaltet werden (Abbildung 1a). Eine geeignete Mischung dieser Magnetfeldgradienten erlaubt eine Drehung der ortskodierten Datenzeilen um das Zentrum des Datenraumes. Damit ergibt sich im Gegensatz zum kartesischen Raster eine radiale Abtastung, bei der die einzelnen Datenpunkte wie auf den Speichen eines Rades angeordnet sind (Abbildung 1b). Diese ursprünglich von Lauterbur eingeführte - und auch im FLASH-Patent (Frahm et al. 1985) als Option beschriebene - Variante war wegen gerätetechnischer Schwierigkeiten in den ersten Jahren der MRT-Entwicklung nahezu vollständig aufgegeben worden. Auch nach Überwindung der verschiedenen Probleme wurde lange Zeit übersehen, dass eine radiale Ortskodierung ohne Phasenkodierung eine Reihe von Vorteilen bietet, die insbesondere für die Echtzeit-MRT genutzt werden können.

Die Bewegungsresistenz der radialen FLASH-Bilder ist so ausgeprägt, dass selbst Bewegungen, die erheblich schneller als die Gesamtmesszeit sind, ohne die aus der kartesischen MRT bekannten Bildfehler dargestellt werden (Abbildung 2). Dabei ist jedoch zu berücksichtigen, dass die Aufnahmen

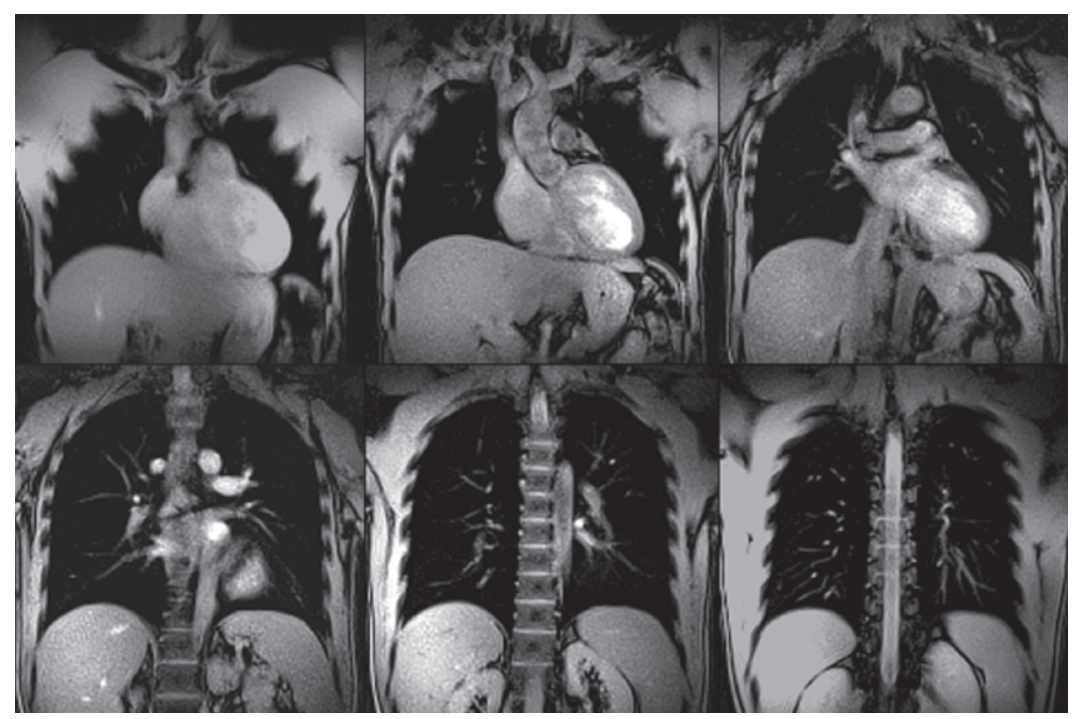

Abbildung 2: MRT-Aufnahmen von Thorax und oberem Abdomen mit der radial kodierten FLASH-Technik. Die Bilder mit einer Messzeit von jeweils 0.5 Sekunden und einer Auflösung von $2 \times 2 \mathrm{~mm}^{2}$ (Schichtdicke $8 \mathrm{~mm}$ ) demonstrieren die fehlerfreie Darstellung bewegter Organe. Die 6 koronalen Schichten zeigen das Herz mit den großen Gefäßen sowie die Leber, die Milz, die Nieren, die Wirbelsäule und das Rückenmark. 
einen zeitlichen Mittelwert über die Dauer der Messung repräsentieren, beispielsweise für die Abbildung des Herzens.

\section{Bildrekonstruktion}

Um aus radialen Daten ein Bild zu berechnen, wird der Datenraum zunächst auf ein rechtwinkliges Raster interpoliert, so dass - wie in der konventionellen MRT - eine inverse Fourier-Transformation zur Rekonstruktion des Bildes genutzt werden kann. Allerdings sind anschliessend noch die Auswirkungen der Interpolation im berechneten Bild zu korrigieren. Für diese als gridding bekannte, etwas aufwendigere Rekonstruktion als in der kartesischen MRT stehen seit vielen Jahren etablierte Algorithmen zur Verfügung (O'Sullivan 1985; Jackson et al. 1991), die auf den heutigen Computern zu keiner verzögerten Darstellung der Bilder oder einer Minderung der Bildqualität führen.

In der Tat zeichnet sich die radiale Ortskodierung neben der Bewegungsresistenz durch weitere vorteilhafte Eigenschaften aus, die die EchtzeitMRT günstig beeinflussen. Dies gilt insbesondere für die prinzipielle Gleichwertigkeit der einzelnen Speichen, die jeweils durch den Ursprung des Datenraumes (Nullpunkt) gehen und ihn dadurch auf stets gleiche Weise abtasten. Dies ist aus folgendem Grund von Bedeutung: im Zentrum des Datenraumes sind die tiefen (kleinen) Raumfrequenzen kodiert, die den großen Strukturen und den wesentlichen Signalanteilen des Bildes entsprechen - also beispielsweise auch die grobe Position eines Untersuchungsobjektes wiedergeben. Die äußeren Bereiche des Datenraumes enthalten ergänzend die hohen Raumfrequenzen, die die Details und die Kantenschärfe bestimmen. Während bei einer kartesischen Abtastung die rechtwinklig verschobenen Datenzeilen in der phasenkodierenden Richtung nur einmal pro Gesamtmessung das Zentrum abtasten, liefert die radiale Ortskodierung mit jeder Teilmessung eine kontinuierliche Erneuerung tiefer Raumfrequenzen und ermöglicht daher eine bessere Anpassung der Messdaten an ein bewegtes Objekt.

Um diese Eigenschaft optimal zu nutzen, wird die radiale Ortskodierung in der FLASH-Sequenz so realisiert, dass die vorgesehene Gesamtzahl der Speichen in mehreren Umläufen gemessen wird (Abbildung 1c). Die Berechnung des Bildes aus einer entsprechenden Anzahl von Teildatensätzen bewirkt eine besonders gleichmäßige Wichtung bewegter Signalanteile. Darüber hinaus kann bei einer seriellen Messung von Bildern bereits eine neue Rekonstruktion erfolgen, wenn nur ein Teil der Daten (beispiels- 
weise ein Umlauf) gemessen wurde. Mit dieser sliding window-Technik, die bereits vor 20 Jahren für kartesische Daten vorgeschlagen wurde (Riederer et al. 1988), lassen sich erheblich höhere Bildfrequenzen erzielen als durch eine Abfolge von Bildern, die jeweils in ihrer Gesamtheit gemessen werden müssen.

Schließlich noch ein Wort zu der benötigten Rechenleistung: wenn die Anzahl der rekonstruierten Bilder pro Sekunde beispielsweise 20 betragen soll, dann muss der Rechenvorgang spätestens nach 50 Millisekunden abgeschlossen sein. Für die heute verwendeten Computer ist das bei einem einzelnen Bild kein Problem. Auf der anderen Seite nutzen alle modernen MRT-Systeme Radiofrequenzantennen zur Datenaufnahme (Empfangsspulen), die zur Verbesserung des Signal-Rausch-Verhältnisses aus vielen Einzelelementen bestehen und deren Signale unabhängig voneinander verarbeitet werden müssen. Bei den hier gezeigten Beispielen wurden gleichzeitig 32 Datensätze aufgenommen, die zur Berechnung eines Bildes zusammengeführt werden mussten. Da dieser Faktor jedoch die verfügbare Rechenkapazität für eine ausreichend schnelle Rekonstruktion übersteigt, wird für die Echtzeit-MRT auf ein mathematisches Verfahren zurückgegriffen, dass die 32 unabhängigen Signale vor der Bildberechnung auf ihre wesentlichen Komponenten zurückführt (Buehrer et al. 2007; Huang et al. 2008). So konnte die verwendete Hauptkomponentenanalyse (principal component analysis) die zu verarbeitenden Signale auf 8 bis 10 Komponenten reduzieren, die eine Berechnung und Darstellung der Bilder in Echtzeit sowie ohne Beeinträchtigung der Qualität ermöglichten (Zhang et al. 2009). Dies ist auf die teilweise räumliche Überlagerung der Spulensensitivitäten und eine entsprechende Redundanz in den Daten zurückzuführen.

\section{Zwei Beispiele}

Die aktuellen Möglichkeiten der Echtzeit-MRT sollen an zwei Beispielen vorgestellt werden, die mit der konventionellen MRT nicht realisierbar sind. Das erste Beispiel betrifft Vorstudien zu einer umfangreichen phonetischen Arbeit, die sich erstmalig mit einer Darstellung derjenigen Sprechbewegungen beschäftigen wird, die bei der Aussprache von Phonemen und Logatomen benötigt werden (Abbildung 3). Die Untersuchungen sollen in verschiedenen Sprachen zunächst an gesunden Versuchspersonen und später an Patienten mit Sprechstörungen durchgeführt werden.

In der Tat erscheint die Echtzeit-MRT als das einzig praktikable bildgebende Verfahren für phonetische Untersuchungen, da Ultraschall-Techni- 

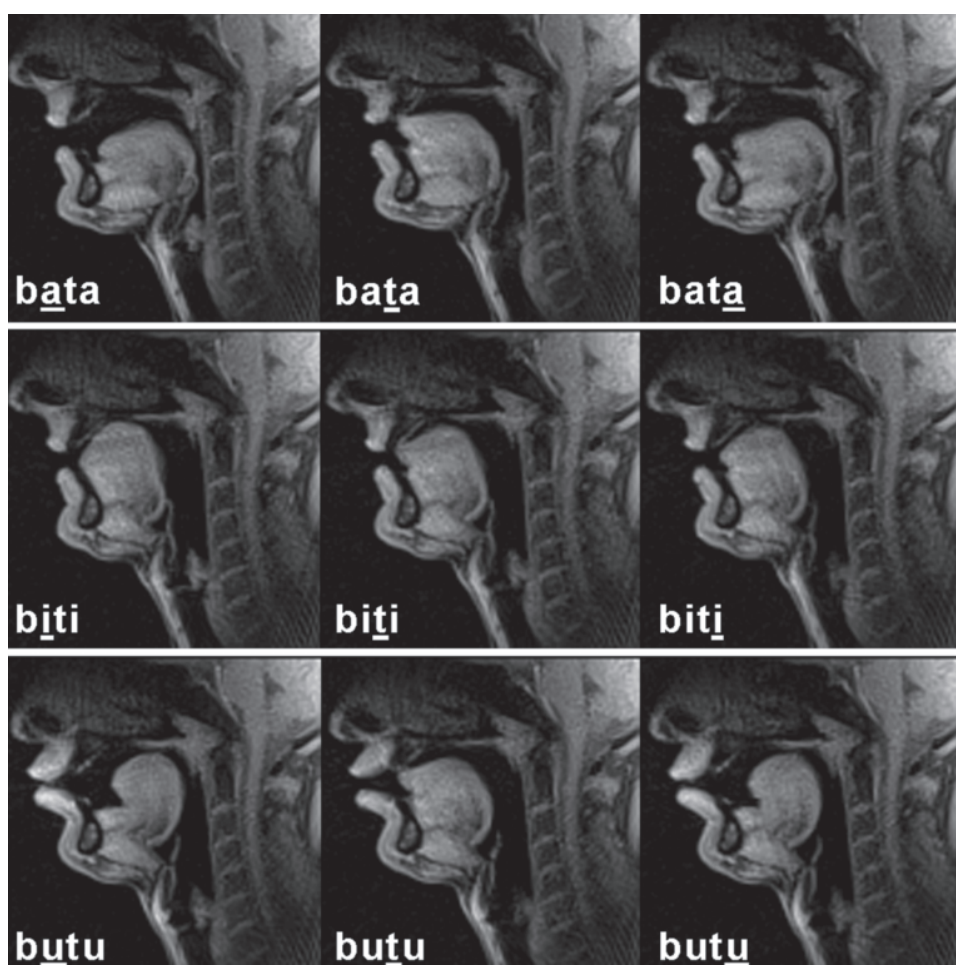

Abbildung 3: MRT-Filme des Sprechvorganges bei den Logatomen [„bata“], [„biti“] und [„,butu“]. Die Einzelbilder aus drei dynamischen Bildserien mit einer zeitlichen Auflösung von 66 Millisekunden (15 Bilder pro Sekunde) entsprechen der Stellung von Lippen, Zunge und Gaumensegel zu den jeweils durch den Unterstrich angegebenen Zeitpunkten, die den Vokalen [,a“], [,"“] und [,u“] bzw. dem Konsonanten „t“ zuzuordnen sind.

ken keine ausreichende räumliche Auflösung bieten, Röntgentechniken zu nicht vertretbaren Strahlenbelastungen führen und endoskopische Ansätze die zu untersuchende Sprechbewegung stören. Als Logatome werden in der Phonetik ein- oder mehrsilbige Wörter bezeichnet, die in der untersuchten Sprache zwar keine Bedeutung besitzen, aber nach den phonetischen und den phonotaktischen Regeln der Sprache richtig gebildet erscheinen. Die hier dargestellten Bewegungen bei den deutschen Logatomen [,bata“], [,biti“] und [„butu“] zeichnen sich vor allem durch markante Unterschiede in der Position der Zunge und der Lippen bei den Vokalen [„a"], [,i“] und [,u"] aus, während eine sehr ähnliche Position der Zunge an der oberen Zahnreihe jeweils dem Konsonanten [,t"] zuzuordnen ist (Abbildung 3). 
Eine wichtige klinische Perspektive für die Echtzeit-MRT wird sich bei der Untersuchung des Herzens ergeben, da das Verfahren auf das für die meisten Herzpatienten schwierige Anhalten des Atems verzichten kann. Da auch - wie bisher üblich - keine Synchronisation der Datenaufnahme mit dem Elektrokardiogramm mehr erforderlich ist, um dynamische Filme des schlagenden Herzens aufzuzeichnen, sollten sich erhebliche Vorteile für die kardiovaskuläre MRT ergeben. Dies setzt allerdings voraus, dass die Aufnahmen eine angemessene räumliche Auflösung und Bildqualität erreichen. Erste Ergebnisse an gesunden Versuchspersonen sind bereits äußerst vielversprechend (Abbildung 4).

Die knappe halbe Sekunde aus dem Echtzeit-Film zeigt das Herz in einem anatomisch definierten Kurzachsenblick. Diese Bildebene repräsentiert einen Schnitt durch die linke Herzkammer senkrecht zu deren Längsachse, so dass ihre Wände eine Kreisform bilden. In dieser Orientierung ist die für den Pumpvorgang des Blutes wichtige systolische Phase des Herzzyklus besonders gut zu erkennen. Sie ist - etwa in der Mitte

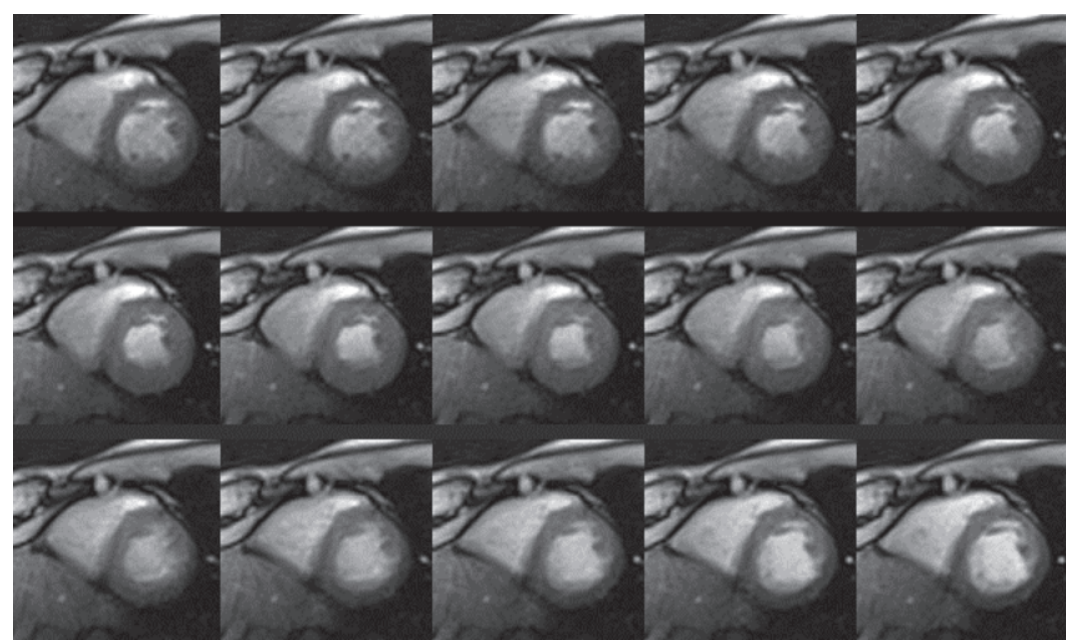

Abbildung 4: MRT-Film des schlagenden Herzens einer gesunden Versuchsperson in Echtzeit bei freier Atmung und ohne Synchronisation mit dem EKG (Kurzachsenblick der linken Herzkammer). Die Abbildung zeigt 15 aufeinanderfolgende Bilder (von links oben nach rechts unten) mit einem Abstand von nur 30 Millisekunden (entsprechend einer Bildfrequenz von 33 Bildern pro Sekunde). Der insgesamt 0.45 Sekunden dauernde Ausschnitt repräsentiert die systolische Phase eines einzelnen Herzschlags, die durch eine Verdickung und Kontraktion des Herzmuskels gekennzeichnet ist. Die helleren Signalanteile entsprechen dem fließenden Blut. 
der Bildsequenz (Abbildung 4) - durch eine maximale Kontraktion und Wandverdickung des Herzmuskels gekennzeichnet.

\section{Ausblick}

Es ist zu erwarten, dass mit der hier vorgestellten Lösung für die EchtzeitMRT eine über die bisher in der Literatur berichteten Versuche hinausgehende, verstärkte klinische und wissenschaftliche Nutzung einsetzen wird. Diese Einschätzung wird auch durch die Tatsache bestärkt, dass das vorgeschlagene Verfahren ohne Schwierigkeit auf den vorhandenen MRTSystemen implementiert werden kann und einer weiten Verbreitung nicht die Notwendigkeit einer gerätetechnischen Umrüstung entgegensteht.

Für medizinische Fragestellungen wird die Echtzeit-MRT vor allem dort Beiträge leisten, wo die zeitliche Auflösung einer Bewegung komplementäre Einsichten zu statischen Aufnahmen verspricht, etwa bei der Untersuchung von Gelenkfunktionen sowie für die kardiovaskuläre Bildgebung. Darüber hinaus eröffnen sich weitergehende Anwendungen für die sogenannte interventionelle MRT, die minimalinvasive Eingriffe durch eine kontinuierliche Bildgebung unterstützen soll. Dazu wird die Echtzeit-MRT mit einer interaktiven Steuerung des MRT-Gerätes kombiniert, um beispielsweise die Schichtposition oder den Bildkontrast bei laufender Messung zu verändern.

In technischer Hinsicht wird an der Beseitigung von verbleibenden Bildfehlern gearbeitet, die bei sehr schnellen Signaländerungen auftreten können. Da radiale Daten stets das ganze Objekt - nur aus einem jeweils anderen Blickwinkel - betrachten, sollten die Signalstärken der einzelnen Datenzeilen für die Bildberechnung vergleichbar sein. Umgekehrt führen Signalschwankungen von Speichen, die bei starken Bewegungen zustandekommen können, zu Inkonsistenzen bei der Bildberechnung und damit zu Bildfehlern. Diese Probleme sind allerdings gut zu identifizieren und sollten daher korrigierbar sein.

Ein anderer Schwerpunkt ist die weitere Verbesserung der zeitlichen Auflösung, die sich insbesondere durch eine noch stärkere Unterabtastung des Datenraumes erhöhen lässt. Dieser Schritt bedeutet jedoch eine völlig neue Bildrekonstruktion, da das bisher verwendete gridding-Verfahren bei einer zu geringen Anzahl von Speichen zu Streifenartefakten in den Bildern führt. Wenn die Daten jedoch nicht mehr ausreichen, um aus ihnen direkt ein Bild zu berechnen, kann man die Bildrekonstruktion als inverses Problem definieren und mit Hilfe numerisch-mathematischer Ansätze eine iterative Schätzung und Optimierung eines Bildes vornehmen, das sowohl zu 
den gemessenen Daten als auch zu bekannten Randbedingungen (a prioriWissen) passt. Obwohl derartige Berechnungen selbst auf modernen Computern erhebliche Rechenzeiten benötigen, konnte bereits gezeigt werden, dass eine Implementierung der Algorithmen auf sogenannten Graphikkarten (graphical processing units) zu erheblichen Beschleunigungen führt, die entsprechende Anwendungen für die Echtzeit-MRT bereits in naher Zukunft realistisch erscheinen lassen (Uecker et al. 2009).

\section{Literatur}

Buehrer M, Pruessmann KP, Boesiger P, Kozerke S. Array compression for MRI with large coil arrays. Magn Reson Med 57:1131-1139, 2007.

Frahm J, Haase A, Matthaei D, Merboldt KD, Hänicke W. Deutsche Patentanmeldung P 3504734.8, 12. Februar 1985.

Frahm J, Haase A, Matthaei D. Rapid NMR imaging of dynamic processes using the FLASH technique. Magn Reson Med 3:321-327, 1986.

Huang F, Vijayakumar S, Li Y, Hertel S, Duensing GR. A software channel compression technique for faster reconstruction with many channels. Magn Reson Imaging 26:133$141,2008$.

Jackson JI, Meyer CG, Nishimura DG. Selection of a convolution function for Fourier inversion using gridding. IEEE Trans Med Imaging 10:473-478, 1991.

Lauterbur PC. Image formation by induced local interactions: Examples employing nuclear magnetic resonance. Nature 242:190-191, 1973.

O'Sullivan JD. A fast sinc function gridding algorithm for Fourier inversion in computer tomography. IEEE Trans Med Imaging 4:200-207, 1985.

Riederer SJ, Tasciyan T, Farzaneh F, Lee JN, Wright RC, Herfkens RJ. MR fluoroscopy: Technical feasibility. Magn Reson Med 8:1-15, 1988.

Uecker M, Zhang S, Frahm J. Nonlinear inverse reconstruction for real-time MRI of the human heart using undersampled radial FLASH. Magn Reson Med, submitted, 2009.

Zhang S. Real-time Magnetic Resonance Imaging. Dissertation, Georg-August-Universität Göttingen, 2009.

Zhang S, Block KT, Frahm J. Magnetic resonance imaging in real time - Advances using radial FLASH. J Magn Reson Imaging, in press, 2009. 